\title{
SYMMETRIC MASSEY PRODUCTS AND A HIRSCH FORMULA IN HOMOLOGY $\left({ }^{1}\right),\left(^{2}\right)$
}

\author{
BY \\ STANLEY O. KOCHMAN
}

\begin{abstract}
A Hirsch formula is proved for the singular chains of a second loop space and is applied to show that the symmetric Massey produce $\langle x\rangle^{p}$ is defined for $x$ an odd dimensional mod $p$ homology class of a second loop space with $p$ an odd prime. $\langle x\rangle^{p}$ is then interpreted in terms of the Dyer-Lashof and Browder operations.
\end{abstract}

1. Introduction. When using the Eilenberg-Moore spectral sequence for odd primes $p$, it is often desirable to interpret the Massey product with a symmetric defining system $\langle x\rangle^{p}, \operatorname{deg} x$ odd, in terms of operations on $x$. We will show that under suitable technical hypotheses, there are operations $\beta Q$ and $\lambda$ defined on the homology of the cobar construction on a differential graded Hopf algebra and $\langle x\rangle^{p}=-\beta Q(x)+\operatorname{ad}_{\lambda}^{p-1}(x)(\beta x)$. We will apply this result to the homology of second and higher loop spaces where we will identify $\beta Q$ with the first nontrivial DyerLashof operation and $\lambda$ with the first Browder operation. Then we will apply this result to the cohomology of any topological space where we will identify $\beta Q$ with the last nontrivial Steenrod operation to obtain a new proof of a theorem of D. Kraines. In our study of the former situation we will show that the singular chains of the Moore loops of a topological monoid satisfy the Hirsch formula

$$
(a b) \cup_{1} c=(-1)^{\operatorname{deg} a} a\left(b \cup_{1} c\right)+(-1)^{\operatorname{deg} b \operatorname{deg} c}\left(a \cup_{1} c\right) b,
$$

which G. Hirsch [8] proved for $a, b$ and $c$ singular cochains on a topological space.

The author is very grateful to J. Peter May for his many helpful suggestions throughout the preparation of this paper.

2. A Hirsch formula in homology. Before we turn our attention to the Hirsch formula, we recall a few definitions.

If $(Y, *)$ is a based topological space then we define the space of loops on $Y$ as in J. Moore [17, p. 18-03] with the convention that if $(r, f) \in \Omega Y$ then $f$ has domain

Received by the editors January 11, 1971 and, in revised form, April 15, 1971.

AMS 1970 subject classifications. Primary 55G30, 55G99, 57F35; Secondary 57F25, 57F30.

Key words and phrases. Symmetric Massey product, Hirsch formula, Eilenberg-Moore spectral sequence, Dyer-Lashof operations, Browder operations, cobar construction.

(1) During part of the preparation of this paper, the author was supported by a National Science Foundation Graduate Fellowship.

$\left.{ }^{2}\right)$ This research is contained in the author's doctoral thesis, submitted to the University of Chicago.

Copyright (C) 1972, American Mathematical Society 
$\boldsymbol{R}$ with $f(t)=*$ if $t \leqq 0$ or $t \geqq r$. If $Y$ is a topological monoid then define the Pontryagin product on the singular chains of $\Omega Y$ to be the composite

$$
C_{k}(\Omega Y) \otimes C_{h}(\Omega Y) \stackrel{\eta}{\longrightarrow} C_{k+h}(\Omega Y \times \Omega Y) \stackrel{P_{*}}{\longrightarrow} C_{k+h}(\Omega Y)
$$

where $\eta$ is the shuffle map and $P\left(\left(r_{1}, f_{1}\right),\left(r_{2}, f_{2}\right)\right)=\left(r_{1}+r_{2}, f\right)$ with $f(t)=f_{1}(t) f_{2}(t)$. $C_{*}(\Omega Y)$ thus becomes a differential Hopf algebra.

Definition 1. Let $A$ be a homotopy commutative DGA-algebra. Any homotopy on $A$ which shows that the product is homotopy commutative is called a cup-one $\left(\cup_{1}\right)$ product. In symbols, for all $a, b \in A$

$$
\partial\left(a \cup_{1} b\right)+\partial(a) \cup_{1} b+(-1)^{\operatorname{deg} a} a \cup_{1} \partial(b)=[a, b] .
$$

Let $\pi=\{e, T\}$ be the cyclic group of order two. The join of $\pi$ with itself is

$$
J^{2} \pi=\{[t x,(1-t) y] \mid t \in[0,1] \text { and } x, y \in \pi\} / \sim
$$

where $\sim$ is the equivalence relation generated by identifying $0 e$ with $0 T$ (see J. Milnor [16, p. 430]). $J^{2} \pi$ has the $\pi$-action

$$
z \cdot[t x,(1-t) y]=[t z x,(1-t) z y] \text { for } x, y, z \in \pi \text { and } t \in[0,1] .
$$

There are singular 1-simplexes $\tau_{1}$ and $\tau_{2}$ on $J^{2} \pi$ defined by $\tau_{1}(t, 1-t)=[(1-t) e, t e]$ and $\tau_{2}(t, 1-t)=[(1-t) T, t e]$ for $t \in[0,1]$. Let $\tau=\tau_{2}-\tau_{1} \in C_{*}\left(J^{2} \pi\right)$.

Definition 2 (E. DYeR AND R. LAShOF [5, p. 37]). If $Y$ is a topological monoid and $X=\Omega Y$ then the following composite defines a cup-one product on the singular chains of $X$ :

$$
\begin{gathered}
C_{*}(X) \otimes C_{*}(X) \stackrel{\iota}{\longrightarrow} C_{*}\left(J^{2} \pi\right) \otimes_{\pi} C_{*}(X) \otimes C_{*}(X) \stackrel{1 \otimes \eta}{\longrightarrow} C_{*}\left(J^{2} \pi\right) \otimes_{\pi} C_{*}(X \times X) \\
\stackrel{\eta}{\longrightarrow} C_{*}\left(J^{2} \pi \times_{\pi} X \times X\right) \stackrel{\theta_{*}}{\longrightarrow} C_{*}(X)
\end{gathered}
$$

where $\iota$ is the inclusion map of degree one defined by $\iota(a \otimes b)=\tau \otimes a \otimes b, \eta$ is the shuffle map and $\theta: J^{2} \pi \times X \times X \rightarrow X$ is the $\pi$-equivariant map defined by

$$
\theta\left([(1-t) x, t e],\left(r_{0}, f_{0}\right),\left(r_{1}, f_{1}\right)\right)(s)=f_{x(0)}^{t}(s) f_{x(1)}^{t}(s),
$$

$f_{0}^{t}=f_{0}, f_{1}^{t}(s)=f_{1}\left(s-t r_{0}\right), x \in \pi, t \in[0,1],\left(r_{0}, f_{0}\right) \in X,\left(r_{1}, f_{1}\right) \in X$ and $s \in\left[0, r_{0}+r_{1}\right]$. Note that $X$ is given the trivial $\pi$-action while $X \times X$ and $C_{*}(X) \otimes C_{*}(X)$ have a $\pi$-action by permuting their factors.

The object of this section is to prove the following theorem:

THEOREM 3. If $a, b$ and $c$ are singular chains on the Moore loops of a topological monoid and $\cup_{1}$ denotes the cup-one product of $E$. Dyer and $R$. Lashof [5] then

$$
\text { (ab) } \cup_{1} c=(-1)^{\operatorname{deg} a} a(b \cup 1 c)+(-1)^{\operatorname{deg} b \cdot \operatorname{deg} c}(a \cup 1 c) b
$$


The following definition and theorem from S. Eilenberg and S. Mac Lane [7] will be used in the proof of Theorem 3 to manipulate the shuffle product.

Definition 4. (a) Let $\Sigma_{n}$ be the symmetric group on $n$ letters which acts on the set $\{0, \ldots, n-1\}$. Then

$\operatorname{sh}\left(n_{1}, \ldots, n_{t}\right)$

$$
=\left\{\sigma \in \Sigma_{n} \mid \sigma(u)<\sigma(v) \text { if } \sum_{i=1}^{j-1} n_{i} \leqq u<v<\sum_{i=1}^{j} n_{i} \text { for some } 1 \leqq j \leqq t\right\}
$$

where $\sum_{i=1}^{t} n_{i}=n$ and $n_{i} \geqq 0,1 \leqq i \leqq t$.

(b) Let $X$ be a topological space. For $1 \leqq j \leqq t$ define the natural degeneracy operators $\rho_{j}: \operatorname{sh}\left(n_{1}, \ldots, n_{t}\right) \rightarrow \operatorname{Hom}\left(C_{n j}(X), C_{n}(X)\right)$ as follows: Let

$$
\sigma \in \operatorname{sh}\left(n_{1}, \ldots, n_{t}\right)
$$

and let

$$
\left\{m_{1}, \ldots, m_{n-n_{j}}\right\}=\{0, \ldots, n-1\}-\left\{\sigma\left(h+\sum_{i=1}^{j-1} n_{j}\right) \mid 0 \leqq h<n_{j}\right\}
$$

with $m_{k}<m_{k+1}$ for $1 \leqq k<n-n_{j}$. Then

$$
\rho_{j}(\sigma)=S_{m_{n-n_{j}}} \circ \cdots \circ S_{m_{1}} .
$$

THEOREM 5. Let $X_{1}, \ldots, X_{t}$ be topological spaces. Define the shuffle map

$$
\eta_{t}:\left[C_{*}\left(X_{1}\right) \otimes \cdots \otimes C_{*}\left(X_{t}\right)\right]_{n} \rightarrow C_{n}\left(X_{1} \times \cdots \times X_{t}\right)
$$

by

$$
\eta_{t}\left(a_{1} \otimes \cdots \otimes a_{t}\right)=\sum_{\sigma \varepsilon \operatorname{sh}\left(n_{1}, \ldots, n_{t}\right)}(\operatorname{sgn} \sigma)\left[\rho_{1}(\sigma)\left(a_{1}\right)\right] \times \cdots \times\left[\rho_{t}(\sigma)\left(a_{t}\right)\right]
$$

where $a_{\imath} \in C_{n_{i}}\left(X_{i}\right)$ for $1 \leqq i \leqq t$ and $n=\sum_{i=1}^{t} n_{i}$. Then $\eta_{t}$ is a natural chain equivalence. Furthermore,

$$
\eta_{t}\left(a_{1} \otimes \cdots \otimes a_{t}\right)=\sum_{i=2}^{t} \sum_{\sigma \in \operatorname{sh}\left(n_{1}+\cdots+n_{t-1}, n_{i}\right)} \eta_{2}\left(\left(\eta_{2} \cdots \eta_{2}\left(\eta_{2}\left(a_{1} \otimes a_{2}\right) \otimes a_{3}\right) \cdots\right) \otimes a_{t}\right) .
$$

That is, the following diagram commutes:

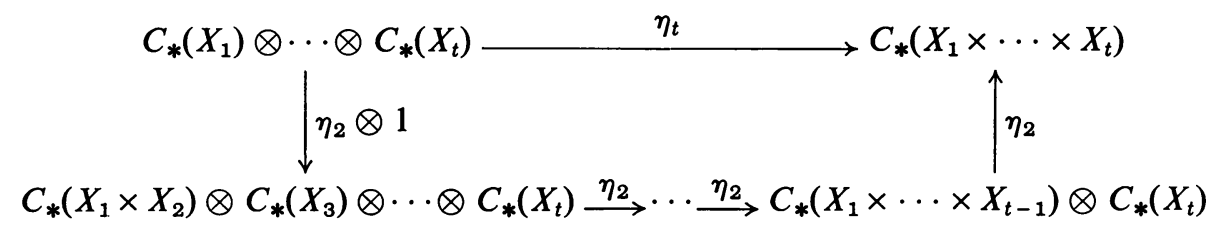

We now apply all of the preceding definitions and Theorem 5 to evaluate $a\left(b \cup_{1} c\right),\left(a \cup_{1} c\right) b$ and $(a b) \cup \cup_{1} c$. 
Lemma 6. Let $Y$ be a topological monoid with $X=\Omega Y$ and $a: \Delta^{\alpha} \rightarrow X, b: \Delta^{\beta} \rightarrow X$ and $c: \Delta^{\gamma} \rightarrow X$ singular simplexes. Then

(i)

$$
\begin{aligned}
& a(b \cup 1 c) \\
& =\sum_{\sigma \in \operatorname{sh}(\alpha, \beta, \gamma, 1)}(-1)^{\beta+\gamma}(\operatorname{sgn} \sigma) P_{*}\left\{\left[\rho_{1}(\sigma)(a)\right] \times \theta_{*}\left[\rho_{4}(\sigma)(\tau) \times \rho_{2}(\sigma)(b) \times \rho_{3}(\sigma)(c)\right]\right\}, \\
& \left(a \cup_{1} c\right) b=\sum_{\sigma \in \operatorname{sh}(\alpha, \beta, \gamma, 1)}(-1)^{\alpha+\beta+\gamma+\beta \gamma}(\operatorname{sgn} \sigma) \\
& \cdot P_{*}\left\{\theta_{*}\left[\rho_{4}(\sigma)(\tau) \times \rho_{1}(\sigma)(a) \times \rho_{3}(\sigma)(c)\right] \times\left[\rho_{2}(\sigma)(b)\right]\right\}, \\
& (a b) \cup \cup_{1} c=\sum_{\sigma \in \operatorname{sh}(\alpha, \beta, \gamma, 1)}(-1)^{\alpha+\beta+\gamma}(\operatorname{sgn} \sigma) \\
& \left.\cdot \theta_{*}\left\{\rho_{4}(\sigma)(\tau) \times P_{*}\left[\left(\rho_{1}(\sigma)(a)\right) \times\left(\rho_{2}(\sigma)(b)\right)\right] \times \rho_{3}(\sigma)(c)\right]\right\} .
\end{aligned}
$$

Proof. (i)

$$
a\left(b \cup_{1} c\right)=\left(P_{*} \circ \eta\right) \circ\left(1 \otimes \theta_{*}\right) \circ(1 \otimes \eta) \circ(1 \otimes 1 \otimes \eta) \circ(1 \otimes \iota)[a \otimes(b \otimes c)]
$$

by Definition 2. Hence

$$
\begin{aligned}
& a\left(b \cup_{1} c\right)=\sum_{\mu \in \operatorname{sh}(\alpha, \beta+\gamma+1)} \sum_{\nu \in \operatorname{sh}(1, \beta+\gamma)} \sum_{\xi \in \operatorname{sh}(\beta, \gamma)}(\operatorname{sgn} \mu)(\operatorname{sgn} \nu)(\operatorname{sgn} \xi) \\
& \cdot P_{*}\left\{\left[\rho_{1}(\mu)(a)\right] \times \theta_{*}\left[\rho_{2}(\mu) \circ \rho_{1}(\nu)(\tau)\right.\right. \times \rho_{2}(\mu) \circ \rho_{2}(\nu) \circ \rho_{1}(\xi)(b) \\
&\left.\left.\times \rho_{2}(\mu) \circ \rho_{2}(\nu) \circ \rho_{2}(\xi)(c)\right]\right\}
\end{aligned}
$$

by Definition 4 . Hence

$$
\begin{array}{r}
a\left(b \cup_{1} c\right)=\sum_{\mu \in \operatorname{sh}(\beta+\gamma+1, \alpha)} \sum_{\nu \in \operatorname{sh}(\beta+\gamma, 1)} \sum_{\xi \in \operatorname{sh}(\beta, \gamma)}(-1)^{\alpha \beta+\alpha \gamma+\alpha+\beta+\gamma}(\operatorname{sgn} \mu)(\operatorname{sgn} \nu)(\operatorname{sgn} \xi) \\
\cdot P_{*}\left\{\left[\rho_{2}(\mu)(a)\right] \times \theta_{*}\left[\rho_{1}(\mu) \circ \rho_{2}(\nu)(\tau)\right.\right. \\
\times \rho_{1}(\mu) \circ \rho_{1}(\nu) \circ \rho_{1}(\xi)(b) \\
\left.\left.\times \rho_{1}(\mu) \circ \rho_{1}(\nu) \circ \rho_{2}(\xi)(c)\right]\right\}
\end{array}
$$

since there is a one-to-one correspondence $F: \operatorname{sh}\left(m, n_{1}, \ldots, n_{t}\right) \rightarrow \operatorname{sh}\left(n_{1}, \ldots, n_{t}, m\right)$ with $\operatorname{sgn} F(\sigma)=(-1)^{m\left(n_{1}+\cdots+n_{t}\right)} \operatorname{sgn} \sigma$ defined by

$$
\begin{aligned}
F(\sigma)(k) & =\sigma(k+m) & & \text { if } 0 \leqq k<n_{1}+\cdots+n_{t}, \\
& =\sigma\left(k-n_{1}-\cdots-n_{t}\right) & & \text { if } n_{1}+\cdots+n_{t} \leqq k<n_{1}+\cdots+n_{t}+m .
\end{aligned}
$$

Hence

$$
\begin{aligned}
a(b \cup 1 & c)=\sum_{\sigma \in \operatorname{sh}(\beta, \gamma, 1, \alpha)}(-1)^{\alpha \beta+\alpha \gamma+\alpha+\beta+\gamma}(\operatorname{sgn} \sigma) \\
\cdot & P_{*}\left\{\left[\rho_{4}(\sigma)(a)\right] \times \theta_{*}\left[\rho_{3}(\sigma)(\tau) \times \rho_{1}(\sigma)(b) \times \rho_{2}(\sigma)(c)\right]\right\}
\end{aligned}
$$

by Theorem 5 . Thus,

$$
\begin{aligned}
a(b \cup 1 c)=\sum_{\sigma \in \operatorname{sh}(\alpha, \beta, \gamma, 1)}(-1)^{\beta+\gamma}(\operatorname{sgn} \sigma) & \\
\cdot & P_{*}\left\{\left[\rho_{1}(\sigma)(a)\right] \times \theta_{*}\left[\rho_{4}(\sigma)(\tau) \times \rho_{2}(\sigma)(b) \times \rho_{3}(\sigma)(c)\right]\right\}
\end{aligned}
$$

by using the above one-to-one correspondence $F$ again.

(ii) and (iii) are proved in the same way as (i). 
Proof of Theorem 3. By the previous lemma, it suffices to show that if

$$
\sigma \in \operatorname{sh}(\alpha, \beta, \gamma, 1)
$$

then

$$
\begin{aligned}
\theta_{*}\left\{\rho_{4}(\sigma)(\tau) \times P_{*}\right. & {\left.\left[\left(\rho_{1}(\sigma)(a)\right) \times\left(\rho_{2}(\sigma)(b)\right)\right] \times \rho_{3}(\sigma)(c)\right\} } \\
= & P_{*}\left\{\left[\rho_{1}(\sigma)(a)\right] \times \theta_{*}\left[\rho_{4}(\sigma)(\tau) \times \rho_{2}(\sigma)(b) \times \rho_{3}(\sigma)(c)\right]\right\} \\
& +P_{*}\left\{\theta_{*}\left[\rho_{4}(\sigma)(\tau) \times \rho_{1}(\sigma)(a) \times \rho_{3}(\sigma)(c)\right] \times\left[\rho_{2}(\sigma)(b)\right]\right\} .
\end{aligned}
$$

Since $\tau=\tau_{2}-\tau_{1}$ this follows from the following three identities:

$$
\begin{aligned}
& \begin{aligned}
\theta_{*}\left\{\rho_{4}(\sigma)\left(\tau_{1}\right) \times P_{*}\left[\left(\rho_{1}(\sigma)(a) \times\right.\right.\right. & \left.\left.\left(\rho_{2}(\sigma)(b)\right)\right] \times \rho_{3}(\sigma)(c)\right\} \\
& =P_{*}\left\{\left[\rho_{1}(\sigma)(a)\right] \times \theta_{*}\left[\rho_{4}(\sigma)\left(\tau_{1}\right) \times \rho_{2}(\sigma)(b) \times \rho_{3}(\sigma)(c)\right]\right\} \\
\theta_{*}\left\{\rho_{4}(\sigma)\left(\tau_{2}\right) \times P_{*}\left[\left(\rho_{1}(\sigma)(a)\right)\right.\right. & \left.\left.\times\left(\rho_{2}(\sigma)(b)\right)\right] \times \rho_{3}(\sigma)(c)\right\} \\
& =P_{*}\left\{\theta_{*}\left[\rho_{4}(\sigma)\left(\tau_{2}\right) \times \rho_{1}(\sigma)(a) \times \rho_{3}(\sigma)(c)\right] \times\left[\rho_{2}(\sigma)(b)\right]\right\}, \\
P_{*}\left\{\left[\rho_{1}(\sigma)(a)\right] \times \theta_{*}\left[\rho_{4}(\sigma)\left(\tau_{2}\right)\right.\right. & \left.\left.\times \rho_{2}(\sigma)(b) \times \rho_{3}(\sigma)(c)\right]\right\} \\
& =P_{*}\left\{\theta_{*}\left[\rho_{4}(\sigma)\left(\tau_{1}\right) \times \rho_{1}(\sigma)(a) \times \rho_{3}(\sigma)(c)\right] \times\left[\rho_{2}(\sigma)(b)\right]\right\} .
\end{aligned}
\end{aligned}
$$

We will only prove (1) since the proofs of (2) and (3) are similar to the proof of (1). We will show that the two singular $(\alpha+\beta+\gamma+1)$-simplexes in (1) are the same by evaluating them on a point $\left(t_{0}, \ldots, t_{\alpha+\beta+\gamma+1}\right)$ of $\Delta^{\alpha+\beta+\gamma+1}$ and then by evaluating the resulting two points of $\Omega Y$ on $s \in[0, \infty)$.

$$
\begin{aligned}
\theta_{*}\left\{\rho_{4}(\sigma)\left(\tau_{1}\right) \times P_{*}\left[\left(\rho_{1}(\sigma)(a)\right)\right.\right. & \left.\left.\times\left(\rho_{2}(\sigma)(b)\right)\right] \times \rho_{3}(\sigma)(c)\right\}\left(t_{0}, \ldots, t_{\alpha+\beta+\gamma+1}\right)(s) \\
& =\theta\left[\tau_{1}(\tilde{t}, 1-\tilde{t}), a\left(t_{0}^{\prime}, \ldots, t_{\alpha}^{\prime}\right) \cdot b\left(t_{0}^{\prime \prime}, \ldots, t_{\beta}^{\prime \prime}\right), c\left(t_{0}^{\prime \prime \prime}, \ldots, t_{\gamma}^{\prime \prime}\right)\right](s) \\
& =\left[a\left(t_{0}^{\prime}, \ldots, t_{\alpha}^{\prime}\right)(s)\right]\left[b\left(t_{0}^{\prime \prime}, \ldots, t_{\beta}^{\prime \prime}\right)(s)\right]\left[c\left(t_{0}^{\prime \prime}, \ldots, t_{\gamma}^{\prime \prime}\right){ }^{i}(s)\right]
\end{aligned}
$$

by Definitions 2 and 4 where

$$
\begin{aligned}
& \rho_{1}(\sigma)\left(t_{0}, \ldots, t_{\alpha+\beta+\gamma+1}\right)=\left(t_{0}^{\prime}, \ldots, t_{\alpha}^{\prime}\right), \\
& \rho_{2}(\sigma)\left(t_{0}, \ldots, t_{\alpha+\beta+\gamma+1}\right)=\left(t_{0}^{\prime \prime}, \ldots, t_{\beta}^{\prime \prime}\right), \\
& \rho_{3}(\sigma)\left(t_{0}, \ldots, t_{\alpha+\beta+\gamma+1}\right)=\left(t_{0}^{\prime \prime}, \ldots, t_{\gamma}^{\prime \prime}\right)
\end{aligned}
$$

and

$$
\rho_{4}(\sigma)\left(t_{0}, \ldots, t_{\alpha+\beta+\gamma+1}\right)=(z, 1-\tilde{t}) .
$$

$$
\begin{aligned}
P_{*}\left\{\left[\rho_{1}(\sigma)(a)\right] \times \theta_{*}\left[\rho_{4}(\sigma)\right.\right. & \left.\left.\left(\tau_{1}\right) \times \rho_{2}(\sigma)(b) \times \rho_{3}(\sigma)(c)\right]\right\}\left(t_{0}, \ldots, t_{\alpha+\beta+\gamma+1}\right)(s) \\
& =\left\{a\left(t_{0}^{\prime}, \ldots, t_{\alpha}^{\prime}\right) \cdot \theta\left[\tau_{1}(\tilde{t}, 1-\tilde{t}), b\left(t_{0}^{\prime \prime}, \ldots, t_{\beta}^{\prime \prime}\right), c\left(t_{0}^{\prime \prime}, \ldots, t_{\gamma}^{\prime \prime}\right)\right]\right\}(s) \\
& =\left[a\left(t_{0}^{\prime}, \ldots, t_{\alpha}^{\prime}\right)(s)\right]\left[b\left(t_{0}^{\prime \prime}, \ldots, t_{\beta}^{\prime \prime}\right)(s)\right]\left[c\left(t_{0}^{\prime \prime}, \ldots, t_{\gamma}^{\prime \prime}\right) \dot{t}(s)\right] .
\end{aligned}
$$

These two calculations prove (1).

As in G. Hirsch [8], we have the following corollary to the Hirsch formula. 
CoROllary 7. Assume that $Y$ is a topological monoid and let $x, y \in H_{*}\left(\Omega Y ; Z_{2}\right)$. If $x y=0$ then $\langle x, y, x\rangle$ is defined and

$$
\langle x, y, x\rangle=y Q^{1+\operatorname{deg} x}(x) \text { modulo } x H_{*}\left(\Omega Y ; Z_{2}\right) .
$$

3. Symmetric Massey products. Henceforth $p$ will denote an odd prime.

Definition 8. Let $A$ be a GDA-algebra over $Z_{p}$ with $x \in H_{2 n-1}(A)$. The symmetric Massey product $\langle x\rangle^{k}$ is said to be defined if there exist elements $a_{1}, \ldots$, $a_{k-1} \in A$, called a defining system of $\langle x\rangle^{k}$, such that $\left\{a_{1}\right\}=x$ and $d\left(a_{t}\right)=\sum_{i=1}^{t-1} a_{i} a_{t-i}$ if $2 \leqq t \leqq k-1$. If $\langle x\rangle^{k}$ is defined then it equals the set of all homology classes $\left\{\sum_{i=1}^{k-1} a_{i} a_{k-i}\right\}$ where $a_{1}, \ldots, a_{k-1}$ varies over all defining systems of $\langle x\rangle^{k}$. If $\langle x\rangle^{k}$ is defined and equal to a single homology class then $\langle x\rangle^{k}$ is said to be defined with zero indeterminacy.

LEMMA 9. Let $A$ be a DGA-algebra over $Z_{p}$ which has a cup-one product that satisfies the Hirsch formula. If $x$ is an odd dimensional homology class of $A$ then $\langle x\rangle^{p}$ is defined with zero indeterminacy.

The proof of Lemma 9 is a direct generalization of the proof of Theorems 15 and 17 in D. Kraines [10] where Lemma 9 is proved for the special case when $x$ is an odd dimensional cohomology class.

Definition 10. Let $A$ be a DGA-coalgebra over a commutative ring $R$. Define the cobar construction $F A$ on $A$ as the tensor algebra $T(s I A)$ on the augmentation ideal $s I A$ where the $s$ indicates that the degree of each element is decreased by one. Elements of $T(s I A)$ will be denoted $\left[a_{1}|\cdots| a_{k}\right.$ ] for $a_{1}, \ldots, a_{k} \in s I A$, and [ ] will denote the identity element of $T(s I A)$. $F A$ has three differentials $d, d_{1}$ and $d_{2}$ where

$$
\begin{aligned}
d & =d_{1}+d_{2}, \\
d_{1}\left[a_{1}|\cdots| a_{k}\right] & =\sum_{i=1}^{k}\left[\bar{a}_{1}|\cdots| \bar{a}_{i-1}\left|d\left(a_{i}\right)\right| a_{i+1}|\cdots| a_{k}\right], \\
d_{2}\left[a_{1}|\cdots| a_{k}\right] & =\sum_{i=1}^{k} \sum\left[\bar{a}_{1}|\cdots| \bar{a}_{i-1}\left|\bar{a}_{i}^{\prime}\right| a_{i}^{\prime \prime}\left|a_{i+1}\right| \cdots \mid a_{k}\right],
\end{aligned}
$$

$a_{1}, \ldots, a_{k} \in S I A, \quad \bar{a}_{i}=(-1)^{1+\operatorname{deg} a_{i}} a_{i}$ and $\bar{\psi}\left(a_{i}\right)=\sum a_{i}^{\prime} \otimes a_{i}^{\prime \prime} . \quad F A$ is a differential algebra under $d$. By J. F. Adams [2, p. 36] and J. P. May [14], if $A$ is a differential Hopf algebra then $F A$ has a cup-one product which satisfies the Hirsch formula.

Definition 11, Let $A$ be a connected DGA-Hopf algebra over $Z_{p}$. Define the primitive elements of $A$ by $P A=\{a \in A \mid \psi(a)=a \otimes 1+1 \otimes a\}$. For $a \in A$, let $\bar{\psi}(a)=\psi(a)-a \otimes 1-1 \otimes a$. There is a natural inclusion of complexes $[P A] \rightarrow F A$. When this map induces an isomorphism in homology we will write $H_{*}([P A])$ $=H_{*}(F A)$.

There are two important examples of connected DGA-Hopf algebras $A$ over $Z_{p}$ with $H_{*}([P A])=H_{*}(F A)$. They are $A=C_{*}\left(\Omega S^{2} X\right)$ for $X$ a connected topological space and $A=C^{*}\left(K\left(Z_{p}, n\right)\right), n \geqq 2$. In fact, by D. Kraines [11] these two examples have the stronger property that every decomposable $d_{2}$-cycle of $F A$ is a $d_{2}$-boundary. 
Definition 12. Let $A$ be a connected DGA-Hopf algebra over $Z_{p}$. Define two operations $Q: H_{2 n-1}([P A]) \rightarrow H_{2 n p-1}(F A)$ for $n \geqq 1$ and $\lambda: H_{r}([P A]) \otimes H_{s}([P A])$ $\rightarrow H_{r+s+1}(F A)$ for $r \geqq 0$ and $s \geqq 0$ by $Q\{[a]\}=\left\{\left[a^{p}\right]\right\}, \lambda(1 \otimes x)=\lambda(x \otimes 1)=0$ and $\lambda(\{[b]\} \otimes\{[c]\})=\left\{\left[b c-(-1)^{(r+1)(s+1)} c b\right]\right\}$ where $a \in P A_{2 n}, \quad x \in H_{*}(F A), \quad b \in P A_{r+1}$ with $r>0, c \in P A_{s+1}$ with $s>0, d(a)=0, d(b)=0$ and $d(c)=0$.

We will show in Lemma 15 that $\lambda$ is well defined and if $A=A^{\prime} \otimes Z_{p}$ with $A^{\prime}$ a $Z$-free connected DGA-Hopf algebra then $\beta Q$ is well defined. Thereafter, we will prove the following theorem.

THEOREM 13. Let $A^{\prime}$ be a Z-free connected DGA-Hopf algebra with $A=A^{\prime} \otimes Z_{p}$ and assume that every decomposable $d_{2}$-cycle of $F A$ is a $d_{2}$-boundary. If $x \in H_{2 n-1}(F A)$, $n \geqq 1$, then $\langle x\rangle^{p}$ is defined with zero indeterminacy and

$$
\langle x\rangle^{p}=-\beta Q(x)+\operatorname{ad}_{\lambda}^{p-1}(x)(\beta x) .
$$

Recall the use of the notation $\operatorname{ad}_{\lambda}$ :

$$
\operatorname{ad}_{\lambda}^{1}(x)(y)=\lambda(x \otimes y) \quad \text { and } \quad \operatorname{ad}_{\lambda}^{k}(x)(y)=\lambda\left(x \otimes \operatorname{ad}_{\lambda}^{k-1}(x)(y)\right) \quad \text { if } k \geqq 2 .
$$

Similarly in any algebra over $Z_{p}$ we may define $\operatorname{ad}^{k}(x)(y)$ by substituting the commutator operation for $\lambda$. Thus,

$$
\begin{aligned}
\operatorname{ad}^{p-1}(x)(y) & =[x,[x, \ldots,[x, y] \ldots] \quad(x \text { taken } p-1 \text { times }) \\
& =\sum_{i=0}^{p-1} x^{p-i-1} y x^{i}
\end{aligned}
$$

if $\operatorname{deg} x$ or $\operatorname{deg} y$ is even by N. Jacobson [9, p. 186]. Observe that if $x=\{[a]\}$, $y=\{[b]\}, a, b \in P A, d(a)=0$ and $d(b)=0$ then $\operatorname{ad}_{\lambda}^{p-1}(x)(y)=\left\{\left[\operatorname{ad}^{p-1}(a)(b)\right]\right\}$.

Lemma 14. Let $A$ be a connected DGA-Hopf algebra over $Z_{p}$. If $a \in P A_{2 n}$ with $d(a)=0$ then $\langle\{[a]\}\rangle^{p} \in H_{2 n p-2}(F A)$ is defined with zero indeterminacy and

$$
\langle\{[a]\}\rangle^{p}=\left\{\sum_{i=1}^{p-1} \frac{(i, p-i)}{p}\left[a^{i} \mid a^{p-i}\right]\right\} .
$$

Proof. By Lemma 9, $\langle\{[a]\}\rangle^{p}$ is defined with zero indeterminacy. Let $a_{i}=$ $(-1)^{i+1}(1 / i !)\left[a^{i}\right]$ if $1 \leqq i \leqq p-1$. Then $a_{1}, \ldots, a_{p-1}$ is a defining system for $\langle\{[a]\}\rangle^{p}$, and

$$
\sum_{i=1}^{p-1} a_{i} a_{p-i}=\sum_{i=1}^{p-1} \frac{(i, p-i)}{p}\left[a^{i} \mid a^{p-i}\right]
$$

Lemma 15. Let $A^{\prime}$ be a $Z$-free connected $D G A$-algebra with $A=A^{\prime} \otimes Z_{p}$. Then $\beta Q$ and $\lambda$ are well defined.

Proof. To prove that $\beta Q$ is well defined, we must show that if $b \in P A_{2 n+1}$, $a \in P A_{2 n}$ and $d(a)=0$ then $\beta\left\{\left[a^{p}\right]\right\}=\beta\left\{\left[(a+d(b))^{p}\right]\right\}$. By N. Jacobson [9, p. 187], $(a+d(b))^{p}-a^{p}-d(b)^{p}$ is in the sub-Lie algebra $S$ of $P A$ generated by $a$ and $d(b)$. Clearly, every element of $S$ except for $Z_{p}$-multiples of $a$ is a boundary. Hence 
$\left[(a+d(b))^{p}\right]$ is homologous to $\left[a^{p}\right]+\left[d(b)^{p}\right]$. Therefore $\beta\left\{\left[(a+d(b))^{p}\right]\right\}=\beta\left\{\left[a^{p}\right]\right\}$ since

$$
\begin{aligned}
\beta\left\{\left[d(b)^{p}\right]\right\} & =-\left\{\sum_{i=1}^{p-1} \frac{(i, p-i)}{p}\left[d(b)^{i} \mid d(b)^{p-i}\right]\right\} \\
& =-\langle\{[d(b)]\}\rangle^{p} \quad \text { by Lemma } 14 \\
& =-\langle\{d[b]\}\rangle^{p}=0 .
\end{aligned}
$$

$\lambda$ is well defined since if $a \in P A_{r}, b \in P A_{s}, c \in P A_{r+1}, e \in P A_{s+1}$ with $r, s \geqq 2, d(a)=0$ and $d(b)=0$ then

$$
\begin{aligned}
& {\left[(a+d(c))(b+d(e))-(-1)^{r s}(b+d(e))(a+d(c))\right]} \\
& \quad=\left[a b-(-1)^{r s} b a\right] \\
& \quad+d\left(\left[c b-(-1)^{r(s+1)} b c\right]+(-1)^{s}\left[a e-(-1)^{s(r+1)} e a\right]+\left[c d(e)-(-1)^{r(s+1)} d(e) c\right]\right) .
\end{aligned}
$$

Proof of Theorem 13. We will compute $\beta Q(x)$ by using the hypothesis that every decomposable $d_{2}$-cycle is a $d_{2}$-boundary and then relate the result to $\langle x\rangle^{p}$ by applying Lemma 14. Since $H_{*}([P A])=H_{*}(F A)$, write $x=\{[a]\}$ with $a \in P A_{2 n}$ and $d(a)=0$. Choose any $a^{\prime} \in A_{2 n}^{\prime}$ which reduces to $a \bmod p$. Then $d\left(a^{\prime}\right)=p b^{\prime}$ and $\bar{\psi}\left(a^{\prime}\right)=p \sum_{i=1}^{t} a_{i}^{\prime} \otimes b_{i}^{\prime}$ for some $b^{\prime}, a_{i}^{\prime}, b_{i}^{\prime} \in A^{\prime}$. Let $b^{\prime}, a_{i}^{\prime}, b_{i}^{\prime}$ reduce mod $p$ to $b, a_{i}, b_{i} \in A$ respectively. Then $\beta\{[a]\}=\left\{[b]+\sum_{i=1}^{t}\left[\bar{a}_{i} \mid b_{i}\right]\right\} . \sum_{i=1}^{t}\left[\bar{a}_{i} \mid b_{i}\right]$ is a decomposable $d_{2^{-}}$ cycle which by hypothesis must bound. Hence there exists $c \in A_{2 n}$ with $\bar{\psi}(c)=$ $\sum_{i=1}^{t} a_{i} \otimes b_{i}$. Thus, $\beta\{[a]\}=\{[b-d(c)]\}$. Choose $c^{\prime} \in A_{2 n}^{\prime}$ which reduces to $c \bmod p$. Let $a^{\prime \prime}=a^{\prime}-p c^{\prime}$ and $b^{\prime \prime}=b^{\prime}-d\left(c^{\prime}\right)$. Then $a^{\prime \prime}$ and $b^{\prime \prime}$ reduce mod $p$ to $a$ and $b$ respectively, $d\left(a^{\prime \prime}\right)=p b^{\prime \prime}$ and $\bar{\psi}\left(a^{\prime \prime}\right)=p^{2} e^{\prime}$ for some $e^{\prime} \in\left(A^{\prime} \otimes A^{\prime}\right)_{2 n}$.

since

$$
d_{1}\left[a^{\prime \prime}\right]=p\left[\operatorname{ad}^{p-1}\left(a^{\prime \prime}\right)\left(b^{\prime \prime}\right)\right]
$$

$$
d\left(a^{\prime \prime}\right)=\sum_{i=1}^{p} a^{\prime \prime i-1} d\left(a^{\prime \prime}\right) a^{\prime p-i}=p \sum_{i=1}^{p} a^{\prime \prime i-1} b^{\prime \prime} a^{\prime p-i}=p \operatorname{ad}^{p-1}\left(a^{\prime \prime}\right)\left(b^{\prime \prime}\right)
$$

by N. Jacobson $[9$, p. 186].

since

$$
d_{2}\left[a^{\prime \prime}\right]=-p \sum_{i=1}^{p-1} \frac{(i, p-i)}{p}\left[a^{\prime \prime} \mid a^{\prime \prime p-i}\right] \text { modulo } p^{2} A^{\prime} \otimes A^{\prime}
$$

$$
\begin{aligned}
\psi\left(a^{\prime \prime}\right) & =\left(a^{\prime \prime} \otimes 1+1 \otimes a^{\prime \prime}+p^{2} e^{\prime}\right)^{p} \\
& =\left(a^{\prime \prime} \otimes 1+1 \otimes a^{\prime \prime}\right)^{p} \quad \text { modulo } p^{2} A^{\prime} \otimes A^{\prime} \\
& =\sum_{i=1}^{p-1}(i, p-i) a^{\prime \prime i} \otimes a^{\prime p-i} \quad \text { modulo } p^{2} A^{\prime} \otimes A^{\prime}
\end{aligned}
$$

Hence

$$
\begin{aligned}
\beta Q(x)=\beta\left\{\left[a^{p}\right]\right\} & =\left\{\left[\operatorname{ad}^{p-1}(a)(b)\right]\right\}-\left\{\sum_{i=1}^{p-1} \frac{(i, p-i)}{p}\left[a^{i} \mid a^{p-i}\right]\right\} \\
& =\operatorname{ad}_{\lambda}^{p-1}(x)(\beta x)-\langle x\rangle^{p}
\end{aligned}
$$

by Lemma 14 and Definition 12 . 
Note that A. Clark [4] contains germs of some of the elements of the proof of Theorem 13.

When we apply Theorem 13 to the homology of a second loop space and to the cohomology of any topological space, the major task will be to identify the operations $\beta Q$ and $\lambda$ with the Dyer-Lashof operation $\beta Q^{n}$ and the first Browder operation $\lambda_{1}$ in homology and with the Steenrod operation $\beta \mathscr{P}^{n}$ and 0 in cohomology. We will first show in Theorem 17 that $\beta Q$ and $\lambda$ have all the properties that one expects. Then under suitable technical hypotheses we will prove a uniqueness theorem for $\beta Q$.

Definition 16. Let $A$ be a connected DGA-Hopf algebra over $Z_{p}$. Define the suspension map $\sigma: I H_{n}([P A]) \rightarrow H_{n+1}(A), n \geqq 0$ by $\sigma\{[a]\}=\{a\}$ if $a \in P A$ with $d(a)=0$. Note that $\sigma$ is well defined, the image of $\sigma$ is a submodule of $P H_{*}(A)$ and if $H_{*}([P A])=H_{*}(F A)$ then $I H_{*}(F A)^{2}$ is contained in the kernel of $\sigma$.

THEOREM 17. Let $\mathscr{C}$ be the category of Z-free connected DGA-Hopf algebras $A^{\prime}$ such that every decomposable $d_{2}$-cycle of $F A$ is a $d_{2}$-boundary where $A=A^{\prime} \otimes Z_{p}$. Then $\beta Q$ and $\lambda$ satisfy the following properties on the objects of $\mathscr{C}$ :

(a) $\lambda$ is natural.

(a)' $\beta Q$ is natural.

(b) $\lambda$ is linear.

(b) $\beta Q(x+y)=\beta Q(x)+\beta Q(y)+\sum_{i=1}^{p-1} \beta d^{i}(x \otimes y)$ and $\beta Q(k x)=k \beta Q(x) \quad$ if $x, y \in H_{2 n-1}(F A)$ and $k \in Z_{p}$ where $d^{i}(x \otimes y)$ is defined by

$$
i d^{i}(x \otimes y)=\sum \operatorname{ad}_{\lambda^{1}}^{j_{1}}(x) \operatorname{ad}_{\lambda^{1}}^{k_{1}}(y) \cdots \operatorname{ad}_{\lambda^{j}}^{j_{r}}(x) \operatorname{ad}_{\lambda^{r}}^{k_{r}}(y)(x),
$$

the sum taken over all $\left(j_{1}, k_{1}, \ldots, j_{r}, k_{r}\right)$ such that $k_{s} \geqq 1, j_{1} \geqq 0, j_{t} \geqq 1$ if $t>1, \sum j_{s}=i-1$ and $\sum k_{s}=p-i$. The important points to note are:

(1) $\beta Q(x+y)-\beta Q(x)-\beta Q(y)$ is in the image under the Bockstein of the sub- $\lambda$ algebra of $H_{*}(F A)$ generated by $x$ and $y$,

(2) by (f), $\beta Q$ is additive if $A$ has a cup-one product such that $a, b \in P A$ implies $a \cup_{1} b \in P A$.

(c) $\lambda$ suspends to the commutator operation on $H_{*}(A)$.

(c)' The relation $Q$ suspends to the pth power operation on $H_{*}(A)$.

(d) (External Cartan Formula) Let $A^{\prime}, B^{\prime}, C^{\prime} \in \mathscr{C}$ and assume that $f^{\prime}: A^{\prime} \otimes B^{\prime}$ $\rightarrow C^{\prime}$ is a map of DGA-Hopf algebras with $f_{*}: H_{*}(A \otimes B) \rightarrow H_{*}(C)$ an isomorphism. Let $\eta: F A \otimes F B \rightarrow F(A \otimes B)$ be the canonical map. Then $\eta_{*}^{-1} \circ F(f)_{*}^{-1}$ defines $\lambda$ on $H_{*}(F A) \otimes H_{*}(F B)$ and

$$
\begin{aligned}
\lambda((x \otimes y) \otimes(u \otimes v))= & (-1)^{\operatorname{deg} y(1+\operatorname{deg} u)} \lambda(x \otimes u) \otimes y v \\
& +(-1)^{\operatorname{deg} u(1+\operatorname{deg} y)} x u \otimes \lambda(y \otimes v)
\end{aligned}
$$

where $x, u \in H_{*}(F A)$ and $y, v \in H_{*}(F B)$. 
(d)" (Internal Cartan Formula). If $A \in \mathscr{C}$ and $x, y, u, v \in H_{*}(F A)$ all have positive degree then

$\lambda(x y \otimes u v)=(-1)^{\operatorname{deg} y(1+\operatorname{deg} u)} \lambda(x \otimes u) y v+(-1)^{\operatorname{deg} u(1+\operatorname{deg} y)} x u \lambda(y \otimes v)$

and

$$
\lambda(y \otimes u v)=\lambda(y \otimes u) v+(-1)^{\operatorname{deg} u \operatorname{deg} v} \lambda(y \otimes v) u .
$$

(d)' (Cartan Formula). Let $A^{\prime}, B^{\prime}, C^{\prime} \in \mathscr{C}$ and assume that $f^{\prime}: A^{\prime} \otimes B^{\prime} \rightarrow C^{\prime}$ [or $g^{\prime}: C^{\prime} \rightarrow A^{\prime} \otimes B^{\prime}$ ] is a map of DGA-Hopf algebras with $f_{*}: H_{*}(A \otimes B) \rightarrow$ $H_{*}(C)\left[g_{*}: H_{*}(C) \rightarrow H_{*}(A \otimes B)\right]$ an isomorphism. Then $\eta_{*}^{-1} \circ F(f)_{*}^{-1}\left[\eta_{*}^{-1} \circ F(g)_{*}\right]$ defines $\beta Q$ on $H_{*}(F A) \otimes H_{*}(F B)$ and $\left[\right.$ if $\lambda=0$ on $H_{*}(F A), H_{*}(F B)$ and $H_{*}(F C)$ then]

$$
\beta Q(x \otimes y)=x^{p} \otimes \beta Q(y)+\beta Q(x) \otimes y^{p}
$$

where $x \in H_{*}(F A), y \in H_{*}(F B)$ and $\operatorname{deg} x \otimes y$ is odd.

(e) $\lambda(x \otimes y)=(-1)^{\operatorname{deg} x \cdot \operatorname{deg} y+\operatorname{deg} x+\operatorname{deg} y} \lambda(y \otimes x)$ if $x, y \in H_{*}(F A)$.

(f) If $A$ has a cup-one product such that $a, b \in P A$ implies $a \cup_{1} b \in P A$ then $\lambda=0$ on $H_{*}(F A)$.

(g) $(-1)^{(1+\operatorname{deg} x)(1+\operatorname{deg} z)} \lambda(x \otimes \lambda(y \otimes z))+(-1)^{(1+\operatorname{deg} y)(1+\operatorname{deg} x)} \lambda(y \otimes \lambda(z \otimes x))+$ $(-1)^{(1+\operatorname{deg} z)(1+\operatorname{deg} y)} \lambda(z \otimes \lambda(x \otimes y))=0$ if $x, y, z \in H_{*}(F A)$.

Proof. (a), (a)', (b), (c), (c)', (e) and (g) are clear from Definitions 12 and 16.

(b) $)^{\prime}$ Write $x=\{[a]\}$ and $y=\{[b]\}$ with $a, b \in P A_{2 n}, d(a)=0$ and $d(b)=0$. Then

$$
\beta Q(x+y)=\beta\left\{\left[(a+b)^{p}\right]\right\}=\beta\left\{\left[a^{p}\right]\right\}+\beta\left\{\left[b^{p}\right]\right\}+\sum_{i=1}^{p-1} \beta\left\{\left[s_{i}(a \otimes b)\right]\right\}
$$

by N. Jacobson [9, p. 187]. $s_{i}(a \otimes b)$ is defined in the same way as $d^{i}(a \otimes b)$ by replacing $\lambda$ with the commutator operation. Hence

$$
\begin{gathered}
\beta Q(x+y)=\beta Q(x)+\beta Q(y)+\sum_{i=1}^{p-1} \beta d^{i}(x \otimes y) . \\
\beta Q(k x)=\beta\left\{\left[k^{p} a^{p}\right]\right\}=k \beta\left\{\left[a^{p}\right]\right\}=k \beta Q(x) .
\end{gathered}
$$

(d) Observe that $F(f)_{*}$ is an isomorphism by the naturality of the EilenbergMoore spectral sequence.

Case 1. $x, y, u$ and $v$ all have positive degree. Let $x=\{[a]\}, y=\{[b]\}, u=\{[c]\}$ and $v=\{[e]\}$ with $a \in P A_{\alpha}, b \in P B_{\beta}, c \in P A_{\gamma}, e \in P B_{\varepsilon}, d(a)=0, d(b)=0, d(c)=0$ and $d(e)=0$. Then $F(f) \circ \eta([a] \otimes[b])=[f(a \otimes 1) \mid f(1 \otimes b)]$ and $F(f) \circ \eta([c] \otimes[e])$ $=[f(c \otimes 1) \mid f(1 \otimes e)]$. Since $[f(a \otimes 1) \mid f(1 \otimes b)]$ and $[f(c \otimes 1) \mid f(1 \otimes e)]$ are decomposable $d_{2}$-cycles, there are $g, h \in C$ so that $[f(a \otimes 1) \mid f(1 \otimes b)]$ is homologous to $[d(g)]$ and $[f(c \otimes 1) \mid f(1 \otimes e)]$ is homologous to $[d(h)]$. Moreover, $d(g)$ 
and $d(h)$ are primitive, $\bar{\psi}(g)=(-1)^{\alpha} f(a \otimes 1) \otimes f(1 \otimes b)$ and $\bar{\psi}(h)=(-1)^{\gamma} f(c \otimes 1)$ $\otimes f(1 \otimes e)$. Hence

$\lambda((x \otimes y) \otimes(u \otimes v))$

$$
\begin{aligned}
=\eta_{*}^{-1} \circ F(f)_{*}^{-1}\{[d(g) d(h)- & \left.\left.(-1)^{(\alpha+\beta+1)(\gamma+\varepsilon+1)} d(h) d(g)\right]\right\} \\
=(-1)^{\gamma(\beta+1)}\left\{\left[a c-(-1)^{\alpha \gamma} c a\right] \otimes[b \mid e]\right\}+(-1)^{\beta(\gamma+1)}\left\{[a \mid c] \otimes\left[b e-(-1)^{\beta \varepsilon} e b\right]\right\} & \\
+\eta_{*}^{-1} \circ F(f)_{*}^{-1}\{d([g d(h)- & \left.(-1)^{(\alpha+\beta+1)(\gamma+\varepsilon+1)} h d(g)\right]-[g \mid h] \\
& +(-1)^{(\alpha+\beta+1)(\gamma+\varepsilon+1)}[h \mid g] \\
& -(-1)^{\alpha+\beta}[f(a \otimes 1) \mid f(1 \otimes b) h] \\
& -(-1)^{\beta(\gamma+\varepsilon+1)+\alpha+\gamma+\varepsilon}[f(a \otimes 1) h \mid f(1 \otimes b)] \\
& -(-1)^{(\alpha+\beta)(\gamma+\varepsilon+1)}[f(c \otimes 1) \mid f(1 \otimes e) g] \\
& -(-1)^{\gamma(\alpha+\beta)}[f(c \otimes 1) g \mid f(1 \otimes e)] \\
& \left.\left.-(-1)^{\beta(\varepsilon+\gamma+1)+\alpha}[f(a c \otimes 1) \mid f(1 \otimes e b)]\right)\right\} \\
=(-1)^{\gamma(\beta+1)} \lambda(x \otimes u) \otimes y v & (-1)^{\beta(\gamma+1)} x u \otimes \lambda(y \otimes v) .
\end{aligned}
$$

Case 2. $x=1$ and $y, u, v$ have positive degree. With the notation of Case 1, $\lambda((1 \otimes y) \otimes(u \otimes v))=\eta_{*}^{-1} \circ F(f)_{*}^{-1}\left\{\left[f(1 \otimes b) d(h)-(-1)^{\beta(\gamma+\varepsilon+1)} d(h) f(1 \otimes b)\right]\right\}$

$$
\begin{aligned}
= & (-1)^{\beta(\gamma+1)}\left\{[c] \otimes\left[b e-(-1)^{\beta \varepsilon} e b\right]\right\} \\
& +\eta_{*}^{-1} \circ F(f)_{*}^{-1}\left\{d\left[(-1)^{\beta} f(1 \otimes b) h-(-1)^{\beta(\gamma+\varepsilon+1)} h f(1 \otimes b)\right]\right\} \\
= & (-1)^{\beta(\gamma+1)} u \otimes \lambda(y \otimes v) .
\end{aligned}
$$

Case 3. $x=1, v=1$ and $y, u$ have positive degree. With the notation of Case 1 , $\lambda((1 \otimes y) \otimes(u \otimes 1))=\eta_{*}^{-1} \circ F(f)_{*}^{-1}\left\{\left[f(1 \otimes b) f(c \otimes 1)-(-1)^{\beta \gamma} f(c \otimes 1) f(1 \otimes b)\right]\right\}$ $=\eta_{*}^{-1} \circ F(f)_{*}^{-1}\left\{\left[(-1)^{\beta \gamma} f(c \otimes b)-(-1)^{\beta \gamma} f(c \otimes b)\right]\right\}=0$.

(d)" To prove the first assertion apply Case 1 of the proof of (d) with $A, B$ and $C$ all equal to $A$ and $F(f) \circ \eta$ replaced by the multiplication $\mu$ of $F A$. The computation which concludes the argument must be appropriately altered to take place in $H_{*}(F A)$ rather than in $H_{*}(F A) \otimes H_{*}(F A)$ since $\mu_{*}$ is not an isomorphism. We now prove the second assertion with the notation of Case 1 of the proof of (d).

$$
\begin{aligned}
\lambda(y \otimes u v) & =\left\{\left[b d(h)-(-1)^{\beta(\gamma+\varepsilon+1)} d(h) b\right]\right\}-\left\{d\left[(-1)^{\beta} b h-(-1)^{\beta(\gamma+\varepsilon+1)} h b\right]\right\} \\
& =\left\{\left[b c-(-1)^{\beta \gamma} c b \mid e\right]\right\}+(-1)^{\beta(\gamma+1)}\left\{\left[c \mid b e-(-1)^{\beta \varepsilon} e b\right]\right\} \\
& =\lambda(y \otimes u) v+(-1)^{(\gamma+1)(\varepsilon+1)} \lambda(y \otimes v) u .
\end{aligned}
$$

(d)' We will show that if $\operatorname{deg} x$ is even and $\operatorname{deg} y$ is odd then $\beta Q(x \otimes y)=$ $x^{p} \otimes \beta Q(y) . \xi: F(A \otimes B) \rightarrow F A \otimes F B$ and $F(f): F(A \otimes B) \rightarrow F C$ [or $F(g): F C \rightarrow$ $F(A \otimes B)$ ] are maps of DGA-algebras which induce isomorphisms in homology. Hence by J. P. May [13, p. 540] any one of $F A \otimes F B, F(A \otimes B)$ or $F C$ can be used to calculate Massey products in $H_{*}(F(A \otimes B))$. Let $x=\{[a]\}$ and $y=\{[b]\}$ with $a \in P A, \quad b \in P B, d(a)=0$, and $d(b)=0$. Then $(-1)^{i+1}(1 / i !)[a|\cdots| a] \otimes\left[b^{i}\right]$ for 
$1 \leqq i \leqq p-1$ is a defining system in $F A \otimes F B$ for $\langle x \otimes y\rangle^{p}$. Hence $\langle x \otimes y\rangle^{p}=$ $x^{p} \otimes\langle y\rangle^{p}$.

$$
\begin{aligned}
\beta Q(x \otimes y) & =-\langle x \otimes y\rangle^{p}+\operatorname{ad}_{\lambda}^{p-1}(x \otimes y)(\beta x \otimes y+x \otimes \beta y) \quad \text { by Theorem } 13 \\
& =-x^{p} \otimes\langle y\rangle^{p}+\operatorname{ad}_{\lambda}^{p-1}(x \otimes y)(x \otimes \beta y) \quad \text { by (d) }
\end{aligned}
$$

since $y^{2}=0$ and $\lambda(y \otimes y)=0[\lambda=0]$. Since $\lambda(y \otimes y \beta(y))=y \lambda(y \otimes \beta(y))$ by (d) ${ }^{\prime \prime}$ and $y^{2}=0$,

$\operatorname{ad}_{\lambda}^{p-1}(x \otimes y)(x \otimes \beta y)$

$$
\begin{aligned}
& =x^{p} \otimes \operatorname{ad}_{\lambda}^{p-1}(y)(\beta y)+\sum_{i=1}^{p-1} x^{i-1} \lambda\left(x \otimes x^{p-i}\right) \otimes y \operatorname{ad}_{\lambda}^{p-2}(y)(\beta y) \\
& =x^{p} \otimes \operatorname{ad}_{\lambda}^{p-1}(y)(\beta y)+\sum_{i=1}^{p-1}(p-i) x^{p-2} \lambda(x \otimes x) \otimes y \operatorname{ad}_{\lambda}^{p-2}(y)(\beta y) \quad \text { by }(d)^{\prime \prime} \\
& =x^{p} \otimes \operatorname{ad}_{\lambda}^{p-1}(y)(\beta y)
\end{aligned}
$$

since $\sum_{i=1}^{p-1} p-i=\frac{1}{2} p(p-1)$ which is divisible by $p$. Thus,

$$
\beta Q(x \otimes y)=x^{p} \otimes\left(-\langle y\rangle^{p}+\operatorname{ad}_{\lambda}^{p-1}(y)(\beta y)\right)=x^{p} \otimes \beta Q(y) .
$$

(f) Let $x=\{[a]\}, y=\{[b]\} \in H_{*}(F A)$ with $a, b \in P A, d(a)=0$ and $d(b)=0$. Then $a \cup_{1} b \in P A$ and $d\left(a \cup_{1} b\right)=a b-(-1)^{\operatorname{deg} a \operatorname{deg} b} b a$ by Definition 1. Hence

$$
\lambda(x \otimes y)=\left\{\left[a b-(-1)^{\operatorname{deg} a \operatorname{deg} b} b a\right]\right\}=\{[d(a \cup 1 b)]\}=\left\{d\left[a \cup_{1} b\right]\right\}=0 .
$$

A second loop space $B=\Omega^{2} C$ has two homology operations defined on $H_{*}\left(B ; Z_{p}\right)$ : a Dyer-Lashof operation $Q^{n}: H_{2 n-1}(B) \rightarrow H_{2 n p-1}(B), n \geqq 1$, and a Browder operation $\lambda_{1}: H_{j}(B) \otimes H_{k}(B) \rightarrow H_{j+k+1}(B), j \geqq 0, k \geqq 0$. W. Browder [3] and J. P. May $[15, \S 6]$ have shown that these two operations satisfy the analogues of properties (a)-(g) and (a)'-(d)' of Theorem 17.

The following theorem shows that $\beta Q$ is uniquely determined by several of its properties.

Theorem 18. Let $A^{\prime}, B^{\prime} \in \mathscr{C}$ and assume that $H_{*}(A)=E\left\{L^{-}\right\} \otimes P\left\{L^{+}\right\}$as coalgebras where $L^{-}$is a set of odd degree elements and $L^{+}$is a set of even degree elements. Let $\Delta: A \rightarrow B$ be a map of DGA-Hopf algebras and $f: A \otimes A \rightarrow B$ [or $g: B \rightarrow A \otimes A]$ a map of DGA-Hopf algebras with $f_{*}: H_{*}(A \otimes A) \rightarrow H_{*}(B)[$ or $g_{*}: H_{*}(B) \rightarrow H_{*}(A \otimes A)$ ] an isomorphism [and $\lambda=0$ on $H_{*}(A)$ and on $H_{*}(B)$ ]. Assume that $R: H_{2 n-1}(F X) \rightarrow H_{2 n p-1}(F X)$ for all $n \geqq 1$ is defined for $X=A$ and $X=B$ such that

(1) $R \circ F(\Delta)_{*}=F(\Delta)_{*} \circ R$.

(2) $R(x+y)=R(x)+R(y)+\sum_{i=1}^{p-1} d^{i}(x \otimes y)$ and $R(k x)=k R(x)$ if $k \in Z_{p}$,

$$
x, y \in H_{2 n-1}(F X) \text { for } X=A \text { and } X=B .
$$

(3) $R$ suspends to the pth power operation for $X=A$ and $X=B$.

(4) $R$ satisfies the Cartan formula on $H_{*}(F A) \otimes H_{*}(F A)$.

Then $\beta Q=\beta R$ on $H_{*}(F A)$. 
Proof. Assume that $\beta Q \neq \beta R$ on $H_{*}(F A)$ and let $x \in H_{2 n-1}(F A)$ be an element of smallest degree for which $\beta Q(x) \neq \beta R(x)$. By the naturality of the Eilenberg-Moore spectral sequence $F(f)_{*}: H_{*}(F A) \otimes H_{*}(F A) \rightarrow H_{*}(F B)$ [or $F(g)_{*}: H_{*}(F B) \rightarrow$ $\left.H_{*}(F A) \otimes H_{*}(F A)\right]$ is an isomorphism, and hence we can define a coproduct on $H_{*}(F A)$ by $\psi=F(f)_{*}^{-1} \circ F(\Delta)_{*}\left[\psi=F(g)_{*} \circ F(\Delta)_{*}\right]$. If $\psi(x)=\sum x^{\prime} \otimes x^{\prime \prime}$ then $\psi \circ \beta Q(x)$ $=\sum \beta Q\left(x^{\prime}\right) \otimes x^{\prime \prime}+\sum x^{\prime p} \otimes \beta Q\left(x^{\prime \prime}\right)$ and $\psi \circ \beta R(x)=\sum \beta R\left(x^{\prime}\right) \otimes x^{\prime \prime}+\sum x^{\prime p} \otimes \beta R\left(x^{\prime \prime}\right)$ by Theorem 17(d)' and hypothesis (4) of this theorem. Hence $\beta Q(x)-\beta R(x)$ is primitive. Therefore it is indecomposable since $\operatorname{deg}(\beta Q(x)-\beta R(x))=2 n p-2$, and the only decomposable primitive elements of $H_{*}(F A)$ are $p$ th powers. Let $Q(x)$ denote a fixed choice of the relation $Q$ on $x$. Then $Q(x)-R(x)$ is in the kernel of the suspension map $\sigma$. Furthermore, $Q(x)-R(x)$ is indecomposable because its image under $\beta$ is indecomposable. By J. P. May [12], $H_{*}(F A)=P\left\{s L^{-}\right\} \otimes E\left\{s L^{+}\right\}$ $\otimes P\left\{t L^{+}\right\}$as algebras. Hence $t L^{+}$is a $Z_{p}$-basis of (Kernel $\left.\sigma\right) \cap Q H_{*}(F A)$, all of whose elements lie in degrees congruent to $p-2 \bmod p$. This is a contradiction since $\operatorname{deg}(Q(x)-R(x))=2 n p-1 \not \equiv p-2 \bmod p$. Hence $\beta Q=\beta R$ on $H_{*}(F A)$.

THeOREM 19. Let $x \in H_{2 n-1}(X), X=\Omega^{2} Z, X$ connected and $n \geqq 1$. Then $\langle x\rangle^{p}$ is defined with zero indeterminacy and

$$
\langle x\rangle^{p}=-\beta Q^{n}(x)+\operatorname{ad}_{\lambda_{1}}^{p-1}(x)(\beta x) .
$$

Proof. By Theorem 3 and Lemma $9,\langle x\rangle^{p}$ is defined with zero indeterminacy. The following diagram commutes and the vertical map is a morphism of second loop spaces:

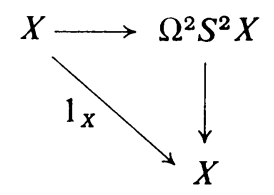

Hence it suffices to evaluate $\langle x\rangle^{p}$ on $H_{*}(X) \subset H_{*}\left(\Omega^{2} S^{2} X\right)$. By J. F. Adams [1], there is a map $\phi: F C_{*}\left(\Omega S^{2} X\right) \rightarrow C_{*}\left(\Omega^{2} S^{2} X\right)$ of differential algebras which induces an isomorphism in homology. By D. Kraines [11], the hypotheses of Theorem 13 are satisfied. Hence $\langle x\rangle^{p}=-\beta Q(x)+\operatorname{ad}_{\lambda}^{p-1}(x)(\beta x)$. It remains to show that $\beta Q$ and $\lambda$ correspond to $\beta Q^{n}$ and $\lambda_{1}$ under the isomorphism $\phi_{*}$. As a first step, we will prove that the Adams map $\phi_{*}$ commutes with the suspension map and the external product. That is, we will show that Figures 1 and 2 commute. Figure 1 clearly commutes. Note that

$$
\eta: C_{*}\left(\Omega S^{2} X\right) \otimes C_{*}\left(\Omega S^{2} Y\right) \rightarrow C_{*}\left(\Omega\left(S^{2} X \times S^{2} Y\right)\right)
$$

is a map of DGA-Hopf algebras and hence induces an algebra homomorphism $F(\eta)$. Figure 3 shows that Figure 2 commutes on elements of $H_{*}\left(F C_{*}\left(\Omega S^{2} X\right)\right) \otimes 1$. Similarly Figure 2 commutes on elements of $1 \otimes H_{*}\left(F C_{*}\left(\Omega S^{2} Y\right)\right)$, and hence Figure 2 commutes on all elements of $H_{*}\left(F C_{*}\left(\Omega S^{2} X\right)\right) \otimes H_{*}\left(F C_{*}\left(\Omega S^{2} Y\right)\right)$ since 


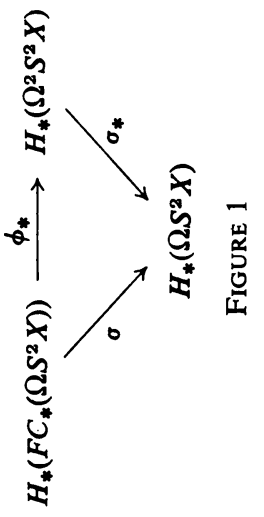

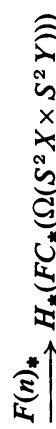

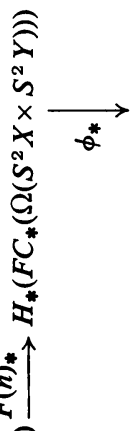

气ิ

$\otimes$

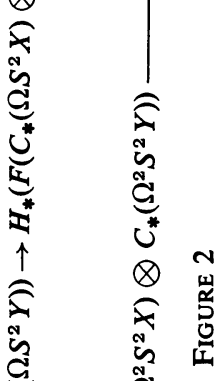

递

$\otimes$

ป

है

$\stackrel{\uparrow}{ร}$

है

:
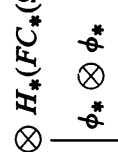

त्र

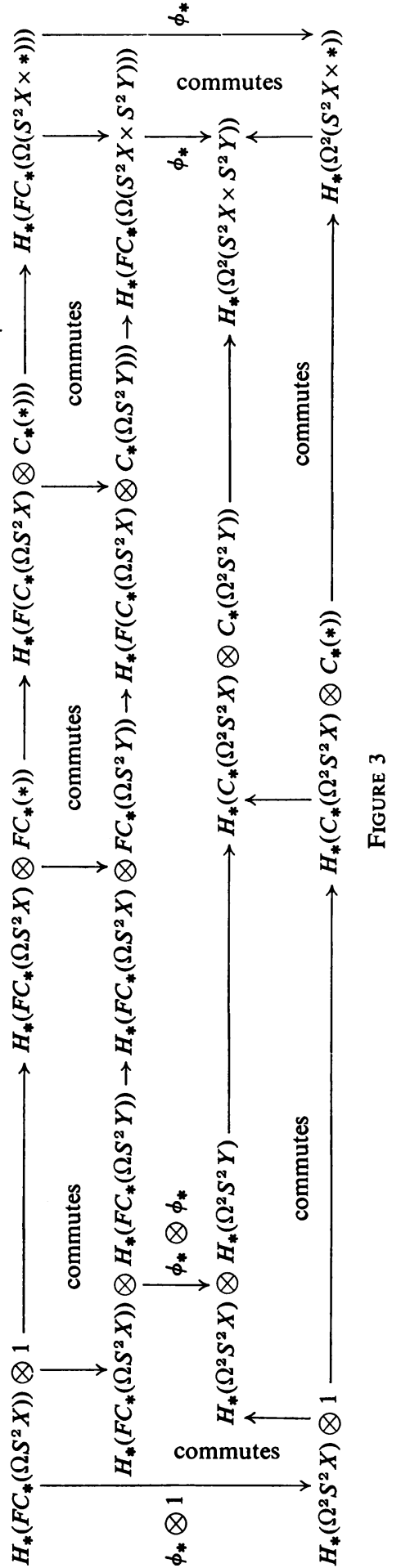


all the maps in Figure 2 are algebra isomorphisms. J. P. May [unpublished] has proved that the first Browder operation $\lambda_{1}$ is the unique operation on the category of second loop spaces which satisfies the analogues of the properties (a)-(g) of Theorem 17. Hence $\phi_{*} \circ \lambda \circ\left(\phi_{*}^{-1} \otimes \phi_{*}^{-1}\right)$ must equal $\lambda_{1}$. Recall that $H_{*}\left(\Omega S^{2} X\right)$ $=T\left(H_{*}(S X)\right)=P\left\{L^{+}\right\} \otimes E\left\{L^{-}\right\}$where $L=L^{+} \cup L^{-}$is the free Lie algebra generated by $H_{*}(S X)$. Hence by Theorem $18, \beta Q$ equals $\phi_{*}^{-1} \circ \beta Q^{n} \circ \phi_{*}$ on $H_{*}\left(\Omega^{2} S^{2} X\right)$.

COROllary 20. Let $x$ be a $(2 n-1)$-dimensional homology class of a connected third loop space, $n \geqq 1$. Then $\langle x\rangle^{p}$ is defined with zero indeterminacy and $\langle x\rangle^{p}$ $=-\beta Q^{n}(x)$.

Proof. This result follows from Theorem 19 and the fact that the first Browder operation is zero on the homology of a third loop space.

Observe that $\operatorname{ad}_{\lambda_{1}}^{p-1}(x)(\beta x)$ may be nonzero. For example, let $M$ be the Moore space with $H_{2 n-1}\left(M ; Z_{p}\right)=Z_{p} x, H_{2 n-2}\left(M ; Z_{p}\right)=Z_{p} \beta x$ and $\tilde{H}_{k}\left(M ; Z_{p}\right)=0$ otherwise, $k \geqq 1$. Then $\operatorname{ad}_{\lambda_{1}}^{p-1}(x)(\beta x) \neq 0$ in $H_{*}\left(\Omega^{2} S^{2} M ; Z_{p}\right)$ since its suspension in $H_{*}\left(\Omega S^{2} M ; Z_{p}\right)$ is $\operatorname{ad}^{p-1}\left(\sigma_{*}(x)\right)\left(\sigma_{*} \beta x\right)$ which is nonzero because $H_{*}\left(\Omega S^{2} M ; Z_{p}\right)$ contains the free Lie algebra generated by $H_{*}\left(S M ; Z_{p}\right)$.

We can also use the machinery of this section to prove the following theorem of D. Kraines [10]:

THEOREM 21. Let $x$ be a $(2 n+1)$-dimensional cohomology class of any topological space, $n \geqq 0$. Then $\langle x\rangle^{p}$ is defined with zero indeterminacy and $\langle x\rangle^{p}=-\beta \mathscr{P}^{n}(x)$.

Proof. $\langle x\rangle^{p}$ is defined with zero indeterminacy by Lemma 9. By the naturality of \langle\rangle$^{p}$ and $\beta \mathscr{P}^{n}$, it suffices to show that $\langle\iota\rangle^{p}=-\beta \mathscr{P}^{n}(\iota)$ where $\iota$ is the fundamental class of $K\left(Z_{p}, 2 n+1\right)$. Assume first that $n \geqq 1$. By S. Eilenberg and S. Mac Lane [6, p. 94] there is a map $\gamma: C^{*}\left(K\left(Z_{p}, 2 n+1\right)\right) \rightarrow F C^{*}\left(K\left(Z_{p}, 2 n\right)\right)$ of differential algebras which induces an isomorphism in homology. By D. Kraines [11] the hypotheses of Theorem 13 are satisfied. Hence $\langle\imath\rangle^{p}=-\beta Q(\iota)+\operatorname{ad}_{\lambda}^{p-1}(\iota)(\beta \iota)$. We will now show that $\lambda=0$. Let $Y$ be a topological space with $u_{i} \in H^{n_{i}}(Y)$ represented by $\hat{u}_{i}: Y \rightarrow K\left(Z_{p}, n_{i}\right)$ for $i=1,2$. In $H^{*}\left(K\left(Z_{p}, n_{1}\right) \times K\left(Z_{p}, n_{2}\right)\right)$,

$$
\lambda\left(\left(\iota_{n_{1}} \otimes 1\right) \otimes\left(1 \otimes \iota_{n_{2}}\right)\right)=0
$$

by Theorem 17(d). Hence $\lambda\left(u_{1} \otimes u_{2}\right)=\left(\hat{u}_{1} \times \hat{u}_{2}\right) * \lambda\left(\left(\iota_{n_{1}} \otimes 1\right) \otimes\left(1 \otimes \iota_{n_{2}}\right)\right)=0$. Thus, $\langle\iota\rangle^{p}=-\beta Q(\iota)$. It remains to show that $\beta Q$ corresponds to $\beta \mathscr{P}^{n}$ under $\gamma_{*}$. Reasoning as in the proof of Theorem 19, one sees that $\gamma_{*}$ commutes with the suspension map and the external product. It is well known that $H^{*}\left(K\left(Z_{p}, 2 n\right)\right)$ is an exterior Hopf algebra on odd degree elements tensored with a polynomial Hopf algebra on even degree elements. Hence by Theorem $18, \beta Q(\iota)=\beta \mathscr{P}^{n}(\iota)$. If $n=0$ then in $H^{*}\left(K\left(Z_{p}, 1\right) \times K\left(Z_{p}, 2\right)\right), \quad\left\langle\iota_{1}\right\rangle^{p} \otimes \iota_{2}^{p}=\left\langle\iota_{1} \otimes \iota_{2}\right\rangle^{p}=-\beta \mathscr{P}^{1}\left(\iota_{1} \otimes \iota_{2}\right)=-\beta \iota_{1} \otimes \iota_{2}^{p}$. Hence $\left\langle\iota_{1}\right\rangle^{p}=-\beta \iota_{1}$. 


\section{BIBLIOGRAPHY}

1. J. F. Adams, On the cobar construction, Proc. Nat. Acad. Sci. U.S.A. 42 (1956), 409-412. MR 18, 59.

2. - On the non-existence of elements of Hopf invariant one, Ann. of Math. (2) 72 (1960), 20-104. MR 25 \#4530.

3. W. Browder, Homology operations and loop spaces, Illinois J. Math. 4 (1960), 347-357. MR 22 \#11395.

4. A. Clark, A homology transgression theorem, Duke Math. J. 35 (1968), 169-173. MR 36 \#3359.

5. E. Dyer and R. K. Lashof, Homology of iterated loop spaces, Amer. J. Math. 84 (1962), 35-88. MR $25 \# 4523$.

6. S. Eilenberg and S. Mac Lane, On the groups of $H(\pi, n)$. I, Ann. of Math. (2) 58 (1953), 55-106. MR 15, 54.

7. - On the groups $H(\pi, n)$. II. Methods of computation, Ann. of Math. (2) 60 (1954), 49-139. MR 16, 391.

8. G. Hirsch, Quelques propriétés des produits de Steenrod, C.R. Acad. Sci. Paris 241 (1955), 923-925. MR 17, 396.

9. N. Jacobson, Lie algebras, Interscience Tracts in Pure and Appl. Math., no. 10, Interscience, New York, 1962. MR 26 \#1345.

10. D. Kraines, Massey higher products, Trans. Amer. Math. Soc. 124 (1966), 431-449. MR 34 \#2010.

11. - Primitive chains and $H_{*}(\Omega X)$, Topology 8 (1969), 31-38. MR 38 \#6581.

12. J. P. May, The cohomology of restricted Lie algebras and of Hopf algebras, J. Algebra 3 (1966), 123-146. MR 33 \#1347.

13. ——, Matric Massey products, J. Algebra 12 (1969), 533-568. MR 39 \#289.

14. - The structure and applications of the Eilenberg-Moore spectral sequence (to appear).

15. - A general algebraic approach to Steenrod operations, Lecture Notes in Math., no. 168, Springer-Verlag, New York, 1970, pp. 153-231.

16. J. W. Milnor, Construction of universal bundles. II, Ann. of Math. (2) 63 (1956), 430-436. MR 17, 1120.

17. J. Moore, Homotopie des complexes monoidaux. I, Séminaire Henri Cartan 1954/55, Secrétariat mathématique, Paris, 1956. MR 19, 439.

Department of Mathematics, University of Chicago, Chicago, Illinois 60637

Department of Mathematics, Yale University, New Haven, Connecticut 06520 